\title{
Assessing the Effectiveness of Proprietary Stormwater Treatment Devices
}

\author{
Matt A. Wilson, John S. Gulliver, Omid Mohseni, and \\ Raymond M. Hozalski
}

Proprietary underground devices are often used as stormwater treatment in dense urban areas due to tight space constraints. Most of these devices remove sediment and other debris from stormwater runoff prior to discharge into lakes, rivers, and streams via the physical separation process of sedimentation. Unfortunately, little is known about the performance of these devices in the field. Evaluation of performance using monitoring studies is problematic because: (i) natural storm events are unpredictable and not reproducible and (ii) it is difficult to obtain representative samples of suspended sediment at both the influent and effluent of the device with automatic samplers. These limitations were overcome in this research by employing a field testing approach. For the testing, a controlled synthetic storm event containing sediment of a fixed size distribution and concentration is fed to a pre-cleaned device. The captured sediment is then removed, sieved, weighed, and the fraction of sediment captured is recorded. The objectives of this research were to: (i) investigate the feasibility and practicality of such a testing approach and (ii) evaluate the effects of sediment size and fluid flow rate on the performance of four devices from different manufacturers. The results of this work are then used to provide a revised sizing criterion that will improve the selection and sizing of these

Wilson, M., J.S. Gulliver, O. Mohseni and R.M. Hozalski. 2008. "Assessing the Effectiveness of Proprietary Stormwater Treatment Devices." Journal of Water Management Modeling R228-14. doi: 10.14796/JWMM.R228-14.

(C) CHI 2008 www.chijournal.org ISSN: 2292-6062 (Formerly in Reliable Modeling of Urban Water Systems. ISBN: 978-0-9808853-0-9) 
devices and their overall performance. The resulting approach, refined through field experiments, will be incorporated into a stormwater best management practice assessment document that will be used by consultants, local governments, and state agencies to assist in selecting, designing, and evaluating stormwater treatment technologies for public infrastructure improvement projects.

\subsection{Introduction}

With the implementation of Phases I and II of the Amendments to the Clean Water Act, states, cities, watershed districts, and other similar entities must manage stormwater discharge. These agencies must determine suitable stormwater best management practices (BMPs) to implement in order to comply with these regulations. Traditionally, detention ponds, bioretention facilities, swales, infiltration basins, and filter strips have been employed to treat stormwater prior to discharge from municipal storm drains, construction sites, and industrial facilities.

An alternative that has become a popular solution for the removal of trash and sediment from stormwater runoff is to use various forms of proprietary underground sedimentation devices. These devices are advantageous in dense urban areas due to their small footprint relative to the facilities above.

Many field monitoring studies have been conducted in recent years to characterize the ability of underground stormwater treatment structures to remove pollutants from stormwater (Waschbusch 1999, Strynchuk et al 2000, England 2001, Yu and Stopinksi 2001, Bonestroo, Rosene, Anderlik and Associates, Inc. 2002 and 2003, ETV 2005a and 2005b, Roseen et al 2005, Fassman 2006). Unfortunately, monitoring studies are problematic for several reasons: (i) natural storm events are unpredictable and not reproducible; (ii) it is difficult to obtain representative samples of suspended sediment at the influent and effluent of the device; and (iii) results of monitoring are typically watershed-specific; that is, it is difficult to apply the results to other watersheds with different land uses, climate, and geology since different hydrology produces different pollutant loads and thus altogether different evaluations. (Wilson 2007).

The main goal of this research was to develop and evaluate a field testing approach for assessing the sediment removal performance of underground proprietary devices. The objectives of this research were to: (i) investigate the feasibility and practicality of such a testing approach and (ii) evaluate the 
effects of sediment size and fluid flow rate on the performance of four devices from different manufacturers. Testing was performed on devices located in Minneapolis, Minnesota and surrounding areas from May to September 2006 and including the following systems: the V2B1 Model 4, by Environment21; the Vortechs Model 2000, by Stormwater360; the Stormceptor STC4800 by Imbrium Systems; and the CDS PMSU20_15 by CDS Technologies.

\subsection{Experimental Approach}

The controlled field testing approach developed and evaluated in this research eliminates the problems involved with sampling a water column for sediments by avoiding sampling the water altogether. Instead of using sampling equipment as a tool in the evaluation of a device, personnel enter the treatment sump to manually remove any solids present, supply artificial runoff using fire hydrant water and a known quantity of sediment from a sediment feeder, then re-enter the treatment area to remove any sediment retained during the test.

Prior to commencement of field testing activities, each of the four test sites required some preparatory steps. Real time discharge measurement capability required the installation of a circular weir and pressure transducer in the storm drain system, as shown in Figure 14.1. A significant cleaning effort was simplified with the assistance of vacuum trucks provided by each city for the initial dewatering and solids removal at each site. A piping system was customized at each site to convey influent test water from a nearby fire hydrant to the structure upstream of the stormwater BMP, as illustrated in Figure 14.2. Finally, sand to be utilized in the artificial runoff for each test was sieved into specific size ranges of interest, with median sizes $107 \mu \mathrm{m}, 303 \mu \mathrm{m}$, and $545 \mu \mathrm{m}$. Figure 14.2 also depicts a stainless steel sediment feeder in the foreground which delivered sand at a known concentration to the test water to produce artificial runoff for passage through the proprietary device.

Wilson et al. (2007) outlined the procedure for field testing which is described briefly here. After the device was dewatered and cleaned, the desired discharge through the system was established. Then, sediment addition to the device influent pipe was initiated by adding equal mass fractions of the three pre-sieved sand sizes using a sediment feeder. At the end of the test, the device was dewatered and the captured sand was removed for drying, sieving and weighing. 


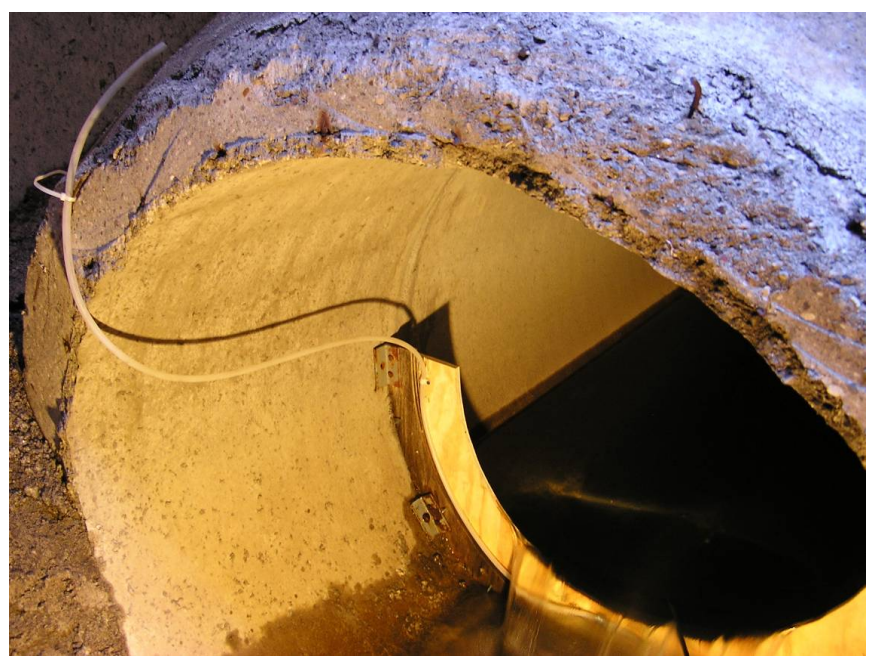

Figure 14.1 A pre-calibrated circular weir, together with a pressure transducer provided real-time discharge data. The weir and transducer were installed in the storm drain at the downstream end of the device.

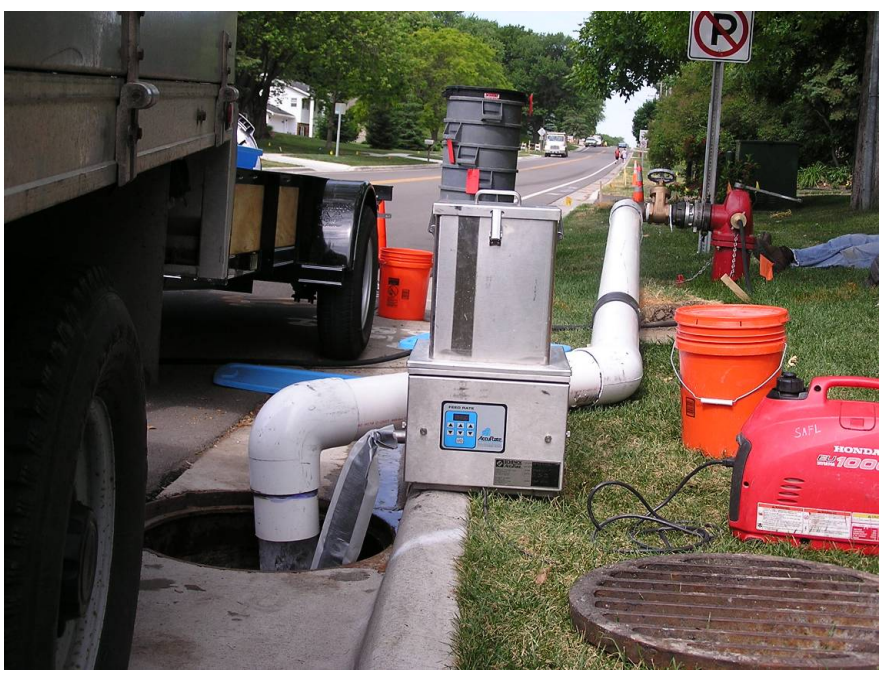

Figure 14.2 A piping system to convey influent test water from the hydrant (back, right) to the structure upstream of the device (front, left). In the foreground is the stainless steel sediment feeder delivering sand. 
In many underground sedimentary devices, pollutants are removed by two treatment chambers. As a result, sand is often retained in both the primary sedimentation chamber as well as the floatables trap, though to a much lesser degree in the floatables chamber. Nevertheless, for accounting purposes, sand retained by the device during testing was collected and inventoried separately for each chamber. The water-sand slurry removed from each treatment chamber sump was oven-dried overnight and re-sieved to determine the amount of mass retained in each size range. This, together with the known quantity of mass delivered, allowed for a removal efficiency calculation. The removal efficiency can be plotted versus a dimensionless parameter to obtain a performance curve for each type of underground structure.

Based upon previous experience with settling of suspended sediment in lakes (Dhamotharan, et al. 1981), the Peclet number $(\mathrm{Pe}=V h / D)$ and a dimensionless time, were shown to have a major influence upon sediment deposition ratio, where $V_{s}$ is particle settling velocity, $h$ is settling depth, and $D$ is the turbulent diffusion coefficient. The Peclet number is defined as a ratio of advection to diffusion, where advective, settling forces are opposed by turbulent diffusion in the system tending to keep a sand particle in suspension. $D$ scales with a velocity times a length scale, where the length scale is the shortest dimension of the flow. In most cases, the length scales of underground structures (diameter $d$ and settling depth $h$ ) are similar, so settling depth $h$ is chosen as the characteristic length for the turbulent diffusion coefficient. Thus $D \sim U h$, and by continuity $D \sim Q h / A$, where $Q$ is discharge. If the vertical cross sectional area $A$ (the direction of mixing) is taken to be $d h$, then $D \sim Q / d$ and $\mathrm{Pe}=V_{s} h d / Q$. The selection of settling depth $h$ as the characteristic length for diffusion is somewhat arbitrary, but allows for both of an underground structure's orthogonal length scales (diameter $d$ and settling distance $h$ ) to contribute to Pe, leading to a more descriptive dimensionless parameter.

\subsection{Results}

Performance curves were developed for each of the four proprietary devices. In all but the Stormceptor device, since it is a single manhole treatment system, multiple performance curves were generated that correspond to removal by the swirling chamber, removal by floatables chamber, and total removal by the combination of both the swirl and floatables chambers. 
The sediment removal performance for the four devices decreased towards zero with decreasing Pe (Figures 14.3 to 14.6). For a given device with fixed length scales $h$ and $d$, a low Pe results from the combination of a small sediment particle settling under the opposition of the mixing energy of high discharges, $Q$. Conversely all four devices removed sediment successfully at higher Pe. For a given device with fixed length scales $h$ and $d$, a high Pe results from the combination of large sand particles settling with minimal opposition from the mixing energy of lower discharges. Ideally, as the length scales become smaller and smaller, toward zero, the removal efficiency would go to zero as well. Conversely, as the length scales are allowed to increase, say toward that greater than a detention pond, the removal efficiency should asymptotically approach 100\%. All four performance curves exhibit an approximately linear relationship (on a semi$\log$ plot) between removal efficiency and Pe, for Pe up to about 3. Again for all four devices tested, at a Pe of approximately 3 , the relationship changes from a relatively steep slope to a near plateau at greater Pe values.

The performance of underground structures is not only dependent on the hydrodynamics inside the system, but is also a function of the storm drain system installed around it. It is likely that these devices would remove nearly all particles above, say $750 \mu \mathrm{m}$, especially at low discharges. This combination of particle size and discharge would produce a relatively high Pe.

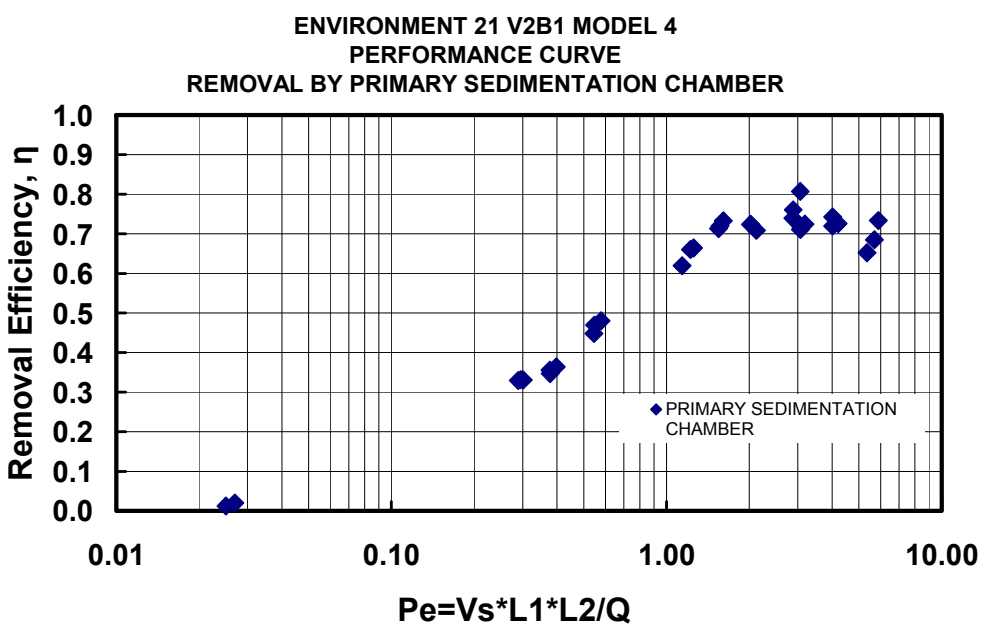

Figure 14.3 Removal efficiency of primary sedimentation chamber of the V2B1 Model 4 versus the Peclet number. 
The measured performance of the Environment21 V2B1 Model 4 is shown in Figure 14.3 as a function of a Peclet number. The performance reflects the mass of material retained in the swirling chamber only. Clearly the results are consistent with the general trends described above. Nevertheless, it appears that at higher Pe, the removal efficiency asymptotically approaches $75 \%$.

The performance of the Vortechs Model 2000 is shown in Figure 14.4, again reflecting only the mass of material retained in the swirling chamber. Unlike the V2B1 device, at mid- to high Pe, the Vortechs removed nearly $100 \%$ of the sediment. It should be stressed, however, that this device was unable to process the manufacturer's maximum treatment flow rate claim of $2.8 \mathrm{cfs}\left(0.08 \mathrm{~m}^{3} \mathrm{~s}\right)$ for the Model 2000 . Rather, the maximum treatment rate as tested was approximately $1.1 \mathrm{cfs}\left(0.03 \mathrm{~m}^{3} \mathrm{~s}\right)$, which corresponded to the flow rate at which influent test water overtopped the bypass weir upstream of the BMP. So, it is possible that this particular device was relatively oversized for the flow rates that could actually be tested, which may explain some of the differences in performance.

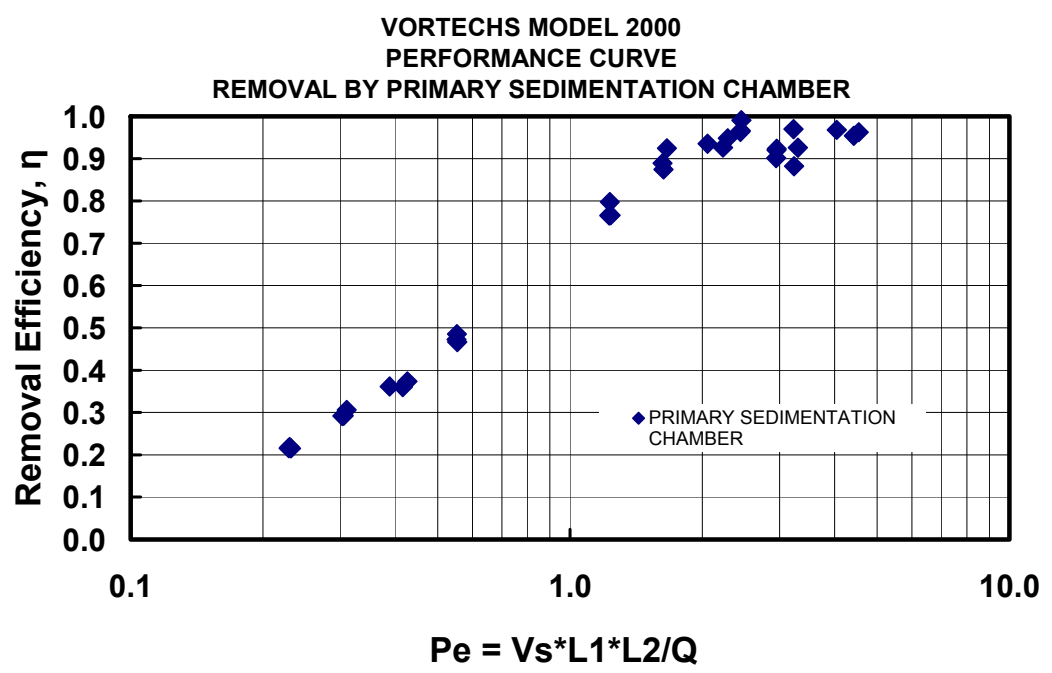

Figure 14.4 Removal efficiency of primary sedimentation chamber of the Vortechs Model 2000 versus the Peclet number.

The performance of the Stormceptor STC4800 is shown in Figure 14.5. Some obvious differences exist between this plot and the others. First, the Pe 
numbers generated during testing are larger, owing to the fact that each of the two length scales, $h$ and $d$, are approximately double that of any other device tested, resulting in an approximate four times difference in the maximum Pe for the Stormceptor. Secondly, it is clear that better removal of the small particles, that is, at Pe less than or equal to approximately 3.0, was achieved by the Stormceptor in comparison with the other devices tested. It seems that the high removal of small particles is due to the large size of the STC4800 (10 ft. $(3.1 \mathrm{~m})$ diameter and $11 \mathrm{ft} .(3.4 \mathrm{~m})$ depth), especially in relation to the maximum treatment rate of the system $\left(1.8 \mathrm{cfs}\right.$ or $\left.0.05 \mathrm{~m}^{3} \mathrm{~s}\right)$. The large size provided a quiescent flow regime near the bottom of the treatment chamber. Additionally, this device was able to remove nearly $100 \%$ of particles in the mid- to upper Pe levels.

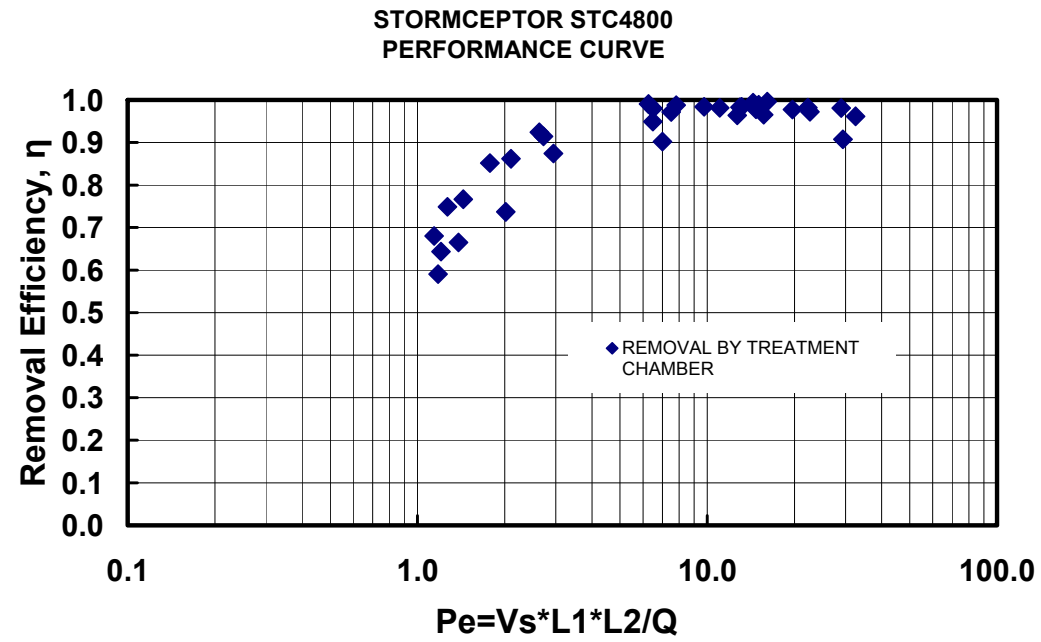

Figure 14.5 Removal efficiency of the Stormceptor STC4800 versus the Peclet number.

Finally, the performance of the CDS PMSU 20_15 is shown in Figure 14.6. This dataset represents only the mass retained in the primary swirling chamber. The general trend of increasing removal with increasing Pe is observed. Nevertheless, there is no obvious plateau like that exhibited by the other three devices at Pe greater than 3.0. Though the maximum removal observed for the CDS was $70 \%$, the trend in performance values suggests complete removal at $\mathrm{Pe}$ numbers much greater than the upper end of the $\mathrm{Pe}$ test range. 


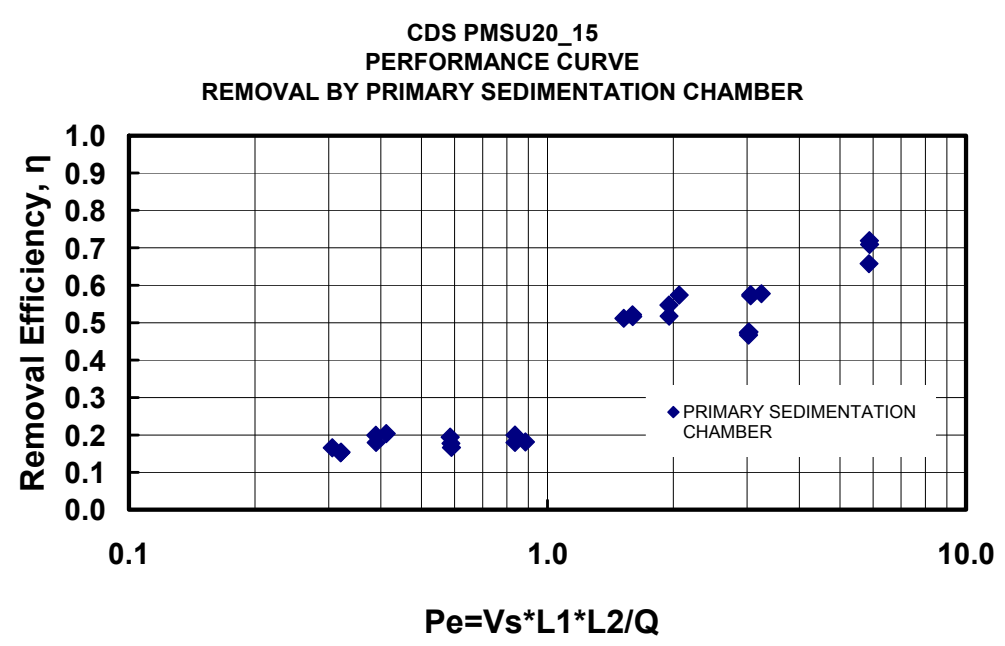

Figure 14.6 Removal efficiency of primary sedimentation chamber of the CDS PMSU20_15 versus the Peclet number,

\subsection{Conclusion}

The removal efficiency of a proprietary underground structure is dependent upon the size of the device, the stormwater discharge, water temperature and the sediment characteristics (i.e. size and density). Thus, in order to correctly predict the expected performance and properly size new installations, it is important to have an understanding of the size distribution of particles in the stormwater runoff from the watershed. This research has developed an accurate testing protocol for underground structures that takes device size, discharge, and sediment size into account, which will be a useful tool for consultants, local governments, and state agencies for selecting, sizing, and evaluating stormwater treatment technologies to protect water resources.

\section{References}

Andoh, R.Y.G. and A.J. Saul (2003). The use of hydrodynamic vortex separators and screening systems to improve water quality. Water Science and Technology, vol 47, no. 4 , pp 175-183. 
Bonestroo, Rosene, Anderlik and Associates, Inc. (2002). Walker Avenue V2B1 Performance. March 19.

Bonestroo, Rosene, Anderlik and Associates, Inc. (2003). Walker Avenue V2B1 20012002 Performance Assessment. April 15.

Dhamotharan, S., et al (1981). Unsteady One-Dimensional Settling of Suspended Sediment. Water Resources Research Vol 17, No 4, p 1125-1132.

England, G. (2001). Success stories of Brevard County, Florida stormwater utility. Journal of Water Resources Planning and Management, vol 127, no. 3, May/June.

Environmental Technology Verification (ETV). (2005a). ETV Report: BaySaver Separation System, model 10k. 05/21/WQPC-WWF, EPA/600/R-05/113, September.

Environmental Technology Verification (ETV). (2005b). ETV Report: Vortechs System, model 1000. 05/24/WQPC-WWF, EPA/600/R-05/140, September.

Fassman, E.A. (2006). Improving effectiveness and evaluation techniques of stormwater best management practices. Journal of Environmental Science and Health Part. 41: 1247-1256.

Guo, Q. (2005). Development of adjustment and scaling factors for measured suspended solids removal performance of stormwater hydrodynamic treatment devices. Proceedings of the World Water and Environmental Resources Congress 2005. Anchorage, AL, May 15-19.

Nnadi, F.N., et al. (2005). Side-by-side evaluation of stormwater proprietary BMPs. Proceedings of the World Water and Environmental Resources Congress 2005. Anchorage, Alaska, May 15-19.

Othmer, E.F. and B.J. Berger. (2002). Future Monitoring Strategies with Lessons Learned on Collecting Representative Samples. Storm Water Program, CSUS Office of Water Programs.

Roseen, R.M., et al. (2005). Normalized technology verification of structural BMPs, Low Impact Development (LID) designs, and manufactured BMPs. Proceedings of the Watershed Management Symposium. Williamsbug, VA.

Schwarz, T. and S. Wells. (1999). Continuous deflective separation of stormwater particulates. Advances in Filtration and Separation Technology.Vol 12, pp 219-226.

Strynchuk, J., et al. (2000). Continuous deflective separation (CDS) unit for sediment control in Brevard County, Florida. Proceedings of the Watershed Management Symposium. Fort Collins, CO.

Waschbusch, R.J. (1999). Evaluation of the effectiveness of an urban stormwater treatment unit in Madison, Wisconsin, 1996-97. U.S.G.S. Water-Resources Investigations Report 99-4195. Middleton, Wisconsin.

Wilson, M, J.S. Gulliver, O. Mohseni, R.M. Hozalski. (2007). Performance assessment of underground stormwater treatment devices. St Anthony Falls Laboratory Project Report No. 494, University of Minnesota, Minneapolis, Minnesota.

$\mathrm{Yu}$, S.L. and M.D.Stopinski. (2001). Testing of ultra-urban stormwater best management practices. VTRC 01-R7, Virginia Transportation Research Council: Charlottesville, VA, 1-43. 